\title{
Steering of a class of nonholonomic systems with drift terms
}

\author{
J.-M. Godhavn ${ }^{\mathrm{a}, *}$, A. Balluchi ${ }^{\mathrm{b}}$, L.S. Crawford ${ }^{\mathrm{c}}$, S.S. Sastry ${ }^{\mathrm{d}}$ \\ a Seatex AS, Pirsenteret, 7005 Trondheim, Norway \\ ${ }^{\mathrm{b}}$ Parades E.E.I.G., Via San Pantaleo, 66, 00186 Rome, Italy \\ c Optimal Synthesis Inc., 450 San Antonio Road, Suite 46, Palo Alto, CA 94306, USA \\ ${ }^{\mathrm{d}}$ Department of Electrical Engineering and Computer Sciences, University of California at Berkeley, \\ Berkeley, CA 94720, USA
}

Received 3 January 1997; revised 22 April 1998; received in final form 16 October 1998

\begin{abstract}
In the present paper nonholonomic systems with drift terms are studied. The discussion is focused on a class of Lagrangian systems with a cyclic coordinate. We present an approach to open-loop path planning in which the system evolution is studied on manifolds of dimension equal to the number of control inputs. A control procedure is derived and it is applied to the planar diver. (C) 1999 Elsevier Science Ltd. All rights reserved.
\end{abstract}

Keywords: Steering; Nonholonomic systems; Drift terms; Path planning; Planar diver; Lagrangian systems; Cyclic coordinates; Bang-bang control

\section{Introduction}

Driftless nonholonomic control systems have been studied in recent years by Walsh and Sastry (1991), Teel et al. (1992), Murray and Sastry (1993), Bloch et al. (1992), Kolmanovsky and McClamroch (1995), and others. Several important results have been derived based on the structure of Lie algebras generated by the control vector fields. A dual point of view using exterior differential systems was developed by Murray (1994), Tilbury et al. (1995), and Tilbury and Sastry (1995). The discussion of nonholonomic systems with drift in the literature has been concentrated on the so-called dynamic extension of drift-free systems by Kapitanovsky et al. (1993) and M'Closkey and Murray (1994). A dynamic extension is an addition of integrators to the velocity inputs. Walsh et al. (1993) have also considered steering on the group of rotations for left-variant systems with drift terms. Kolmanovsky (1995) has treated nonholonomic systems

\footnotetext{
* Corresponding author. Tel. 004773545562 , fax: 004773515020 ; e-mail: jmg@seatex.no.

${ }^{1}$ This paper was not presented at any IFAC meeting. This paper was recommended for publication in revised form by Associate Editor Henu Nijmeijer under the direction of Editor Tamer Başar.
}

with drift terms similar to those in this approach to multibody systems.

An approach to motion planning for nonlinear systems which has received increasing attention in the last few years is the concept of differential flatness (Rouchon et al., 1993). Flatness is locally equivalent to feedback linearizability, and may be used to simplify the design of trajectories. A sufficient condition for flatness is given in Martin (1993) for systems where the dimension of the control vector is one less than the dimension of the state vector. Lagrangian systems of this class where the flat outputs depend on the configuration variables are called configuration flat (Rathinam and Murray, 1996). Controllability properties of Lagrangian systems have also been studied by Lewis and Murray (1995), and dissipative systems by Kelly and Murray (1996).

Classification of nonholonomic systems has also been explored in the literature. A powerful motivation for finding simple standardized forms is to generate reusable control schemes that can be applied to classes of nonlinear control systems. The search for canonical forms by Murray and Sastry (1993) defined the chained form, which has since gained much popularity. This form is equivalent to the power form (Pomet and Samson, 1993). Sinusoids have been used in motion planning for these 
systems because of their smoothness and periodic properties. Systems that can be put in chained form are in a subset of the larger class of nilpotentizable (Di Giamberardino et al., 1996) systems. A system is nilpotent when the Lie algebra is finite dimensional and all Lie brackets of order higher than a finite integer are zero (Kawski, 1988). When the Lie algebra is nilpotent, it is possible to write the solution for the state as a composition of a finite number of solutions to possibly less complicated differential equations. If a system is nilpotent, or nilpotentizable by a diffeomorphic transformation, then Kawski (1993) gives a simple algorithm for transformation to a canonical nonlinear representation of the system. Nilpotent approximations are useful for local stabilization (Hermes et al., 1984). An extension of the derivation of canonical forms to systems with drift terms is a case for further study.

A class of Lagrangian systems with a cyclic coordinate is investigated in this paper as a step toward extending the understanding of control of systems with drift terms. The eventual goal is an increased repertoire of canonical forms. From Noether's Theorem (Arnold 1989) it follows that if a Lagrangian system admits a one parameter group of diffeomorphisms, i.e. there is some kind of symmetry in the system such that the Lagrangian is invariant under some mapping, then a conservation law and a first integral exist. A cyclic coordinate means that the Lagrangian is invariant under, for example, a translation of this coordinate. The first integral will be a conserved generalized momentum conjugate (Goldstein 1980). These systems exhibit a subtriangular structure, as the Jacobian of the vector fields with respect to the state is subtriangular. This structure will be utilized in the design below. If the constant value of this first integral is nonzero, then there is drift in the system. The example considered here is the planar diver. In this example the cyclic coordinate is the body angle, and the generalized momentum is the angular momentum of the diver.

The focus of the present paper is on path planning, the problem of finding inputs that steer the system from an initial state to a desired state. The proposed approach is to consider piecewise constant inputs. In physical systems there is usually an upper bound on how large inputs can be. Further, it is well known that minimum-time control of systems with constrained inputs often results in bang-bang control, in which the control values are at the boundary of the allowed set of inputs. A procedure to find a path that connects a given initial state with a given desired state is presented. The procedure uses bang-bang controls to generate trajectories, and includes both a computation of the maximum and minimum of a scalar function, and a one-dimensional search for a solution. The solution is often not unique. A necessary condition for convergence of the procedure is controllability. Obstacle avoidance is a traditional difficulty that must be considered in motion planning when moving in a cluttered environment. In that case the initial and desired configurations must lie in the same connected component of the free configuration space for the motion planning problem to be solvable (Murray and Sastry, 1993). Obstacle is however not discussed further in this paper.

Open-loop paths are very sensitive to initial condition errors. The combination of an open-loop path planner and an underlying feedback control law for continuous tracking can be seen as the lower modules in a hybrid hierarchical controller scheme for control of nonholonomic mechanical systems as in Varaiya (1993), for example.

The outline of the rest of the paper is as follows. In Section 2 controllability for nonlinear systems with drift terms is considered. The motivating class of nonholonomic systems with drift is derived in Section 3 from the Euler-Lagrange differential equations. The main result of the paper is the path planning procedure given in Section 4.

\section{Controllability}

Consider an affine nonlinear control system

$\dot{\mathbf{x}}=\mathbf{f}(\mathbf{x})+\sum_{i=1}^{m} \mathbf{g}_{i}(\mathbf{x}) u_{i}$

with $\mathbf{u} \in \mathscr{U}$ and $\mathbf{x} \in \mathscr{M}$, under the assumption

Assumption 2.1 (Sussmann, 1987). The state space $\mathscr{M}$ is assumed to be a smooth manifold of dimension n, the control input space $\mathscr{U} \subset \mathbb{R}^{m}$ is assumed compact and convex, the vector fields in the set $\mathscr{F}=\left\{\mathbf{f}, \mathbf{g}_{1}, \ldots, \mathbf{g}_{m}\right\}$ are assumed to be real and analytic, and the input vector fields $\mathbf{g}_{i}$ are assumed to be linearly independent of each other.

We apply the definitions of controllability and accessability by Nijmeijer and van der Schaft (1990), and the Lie algebra rank condition (LARC) and the definition of small time locally controllability (STLC) by Sussmann (1987). For driftless systems, controllability follows from LARC (Nijmeijer and van der Schaft, 1990). Controllability is, however, very different from accessibility for systems with drift.

The following lemma contributed by the authors is a generalization of STLC for systems with nonvanishing drift.

Lemma 2.1. Assume now that the drift term is bounded, but nonzero by $\mathbf{f}(x) \neq 0$, and the vectors $(\operatorname{ad} \mathbf{f})^{k}\left(\mathbf{g}_{i}\right)(\mathbf{x})$, for all $i \in\{1, \ldots, m\}, k \in\{0,1, \ldots\}$ together with the vectors $\left[\mathbf{g}_{i}, \mathbf{g}_{j}\right](\mathbf{x})$, for all pairs $i, j \in\{1, \ldots, m\}$ span $T_{x} \mathscr{M}$. Then system (1) still is $S T L C$ from $\mathbf{x}$ if the controls are sufficiently large, i.e. with controls $\lambda \mathbf{u}$, and 
$\mathbf{u} \in \mathscr{U}=\left\{\left|u_{i}\right| \leq 1, i=1, \ldots, m\right\}$ for some large scalar $\lambda>0$.

Proof. This is the same as STLC but with nonvanishing drift $(\mathbf{f} \neq 0)$

$\frac{\mathrm{d} \mathbf{x}}{\mathrm{d} t}=\mathbf{f}(\mathbf{x})+\lambda \sum_{i=1}^{m} \mathbf{g}_{i}(\mathbf{x}) u_{i}$.

Scaling the time with $\tau=\lambda t$ gives

$\frac{\mathrm{d} \mathbf{x}}{\mathrm{d} \tau}=\frac{1}{\lambda} \mathbf{f}(\mathbf{x})+\sum_{i=1}^{m} \mathbf{g}_{i}(\mathbf{x}) u_{i}$.

Increasing the size of the control inputs is seen to be analogous to reducing the impact of the drift $\mathbf{f}$. From this it can be concluded that if all directions are spanned by these brackets, then large controls will be able to compensate for the bounded drift term, and the system is STLC. The vector fields to consider with large controls are $\mathbf{f}, \lambda \mathbf{g}_{i}, \lambda(\operatorname{ad} \mathbf{f})^{k}\left(\mathbf{g}_{i}\right)$, and $\lambda^{2}\left[\mathbf{g}_{i}, \mathbf{g}_{j}\right]$, and it is seen that the magnitude of all these except $\mathbf{f}$ increase with increasing $\lambda$.

The concept of STLC with unconstrained inputs was introduced by Aeyels (1984).

\section{A class of nonholonomic systems with drift terms}

In this section a motivating class of systems for the discussion later in the paper is presented. The problem caused by the drift term which makes this class different from driftless systems, is considered.

Consider a conservative mechanical system with Lagrangian

$L=L(\mathbf{x}, \dot{\mathbf{x}})=K(\mathbf{x}, \dot{\mathbf{x}})-U(\mathbf{x})$,

where $\mathbf{x} \in \mathbb{R}^{n}$ are the generalized coordinates, $K=$ $\frac{1}{2} \sum_{i, j=1}^{n} a_{i j}(\mathbf{x}) \dot{x}_{i} \dot{x}_{j}$ is the kinetic energy and $U$ is the potential energy. Assume there is a cyclic coordinate, which without loss of generality, is chosen to be $x_{n}$, i.e.

$\frac{\partial L}{\partial x_{n}}=0$.

If no generalized forces act in the direction of $x_{n}\left(Q_{n}=0\right)$, then the Euler-Lagrange differential equations (Goldstein, 1980) yield

$\frac{\mathrm{d}}{\mathrm{d} t}\left(\frac{\partial L}{\partial \dot{x}_{n}}\right)=0$.

This is a special result of Noether's Theorem (Arnold, 1989). A first integral $\mu$ of the motion of the mechanical system is given by

$\mu=\frac{\partial L}{\partial \dot{x}_{n}}=\sum_{i=1}^{n} a_{i n}(\mathbf{x}) \dot{x}_{i}$,

where for all $i \in\{1, \ldots, n\}$

$\frac{\partial a_{i n}(\mathbf{x})}{\partial x_{n}}=0$.

Collect the first $(n-1)$ coordinates in a vector defined by

$\mathbf{x}_{a}=\left[\begin{array}{c}x_{1} \\ \vdots \\ x_{n-1}\end{array}\right]$.

The vector $\mathbf{x}_{a}$ is assumed to be controlled directly by the control inputs $\mathbf{u}=\left[u_{1}, \ldots, u_{n-1}\right]^{\mathrm{T}}$ through single integrators, and the trajectories are designed so that $a_{n n}\left(\mathbf{x}_{a}\right) \neq 0$, then a control system

$\dot{\mathbf{x}}_{a}=\mathbf{u}$,

$\dot{x}_{n}=\frac{\mu}{a_{n n}\left(\mathbf{x}_{a}\right)}-\sum_{i=1}^{n-1} \frac{a_{i n}\left(\mathbf{x}_{a}\right)}{a_{n n}\left(\mathbf{x}_{a}\right)} u_{i}$

is associated to the first integral (7). Define the vector fields $\mathbf{f}, \mathbf{g}_{i}: \mathscr{M} \mapsto \mathbb{R}^{n}, i \in\{1, \ldots, n-1\}$

$\mathbf{f}=\left[\begin{array}{c}0 \\ \vdots \\ \vdots \\ \vdots \\ 0 \\ f\end{array}\right], \quad g_{1}=\left[\begin{array}{c}1 \\ \vdots \\ 0 \\ \vdots \\ 0 \\ g_{1}\end{array}\right], \ldots$,

$\mathbf{g}_{i}=\left[\begin{array}{c}0 \\ \vdots \\ 1 \\ \vdots \\ 0 \\ g_{i}\end{array}\right], \ldots, \quad \mathbf{g}_{n-1}=\left[\begin{array}{c}0 \\ \vdots \\ 0 \\ \vdots \\ 1 \\ g_{n-1}\end{array}\right]$,

where 1 is the $i$ th entry of $\mathbf{g}_{i}$, and with

$f\left(\mathbf{x}_{a}\right)=\frac{\mu}{a_{n n}\left(\mathbf{x}_{a}\right)}$,

$g_{i}\left(\mathbf{x}_{a}\right)=-\frac{a_{i n}\left(\mathbf{x}_{a}\right)}{a_{n n}\left(\mathbf{x}_{a}\right)}$.

The velocities $u_{i}$ are assumed to be controlled by a fast outer loop from generalized forces $\tau_{i}=\dot{u}_{i}$.

Proposition 3.1. System (10) is accessible and STLC for $\mathbf{x} \in \mathscr{M}$ if the controls are sufficiently large, and either from Eqs. (12) and (13), $\partial f / \partial x_{i} \neq 0$ for some $i \in\{1, \ldots, n-1\}$, or 
$\left(\partial g_{j} / \partial x_{i}\right)-\left(\partial g_{i} / \partial x_{j}\right) \neq 0$ for a pair $i, j \in\{1, \ldots, n-1\}$ for all $\mathbf{x} \in \mathscr{M}$.

Proof. The Lie brackets are given by

$$
\left[\mathbf{f}, \mathbf{g}_{i}\right]=\left[\begin{array}{c}
0 \\
\vdots \\
0 \\
-\frac{\partial f}{\partial x_{i}}
\end{array}\right], \quad\left[\mathbf{g}_{i}, \mathbf{g}_{j}\right]=\left[\begin{array}{c}
0 \\
\vdots \\
0 \\
\frac{\partial g_{j}}{\partial x_{i}}-\frac{\partial g_{i}}{\partial x_{j}}
\end{array}\right]
$$

using the fact that the partial of $\mathbf{f}$ and $\mathbf{g}_{i}(11)$ with respect to $x_{n}$ is zero. If one of these Lie brackets (14) is nonzero, then together with all $\mathbf{g}_{i}, i \in\{1, \ldots, n-1\}$, it spans all $\mathbb{R}^{n}$ and system (1) is accessible. Lemma 2.1 ensures STLC provided that the controls are sufficiently large.

\subsection{Case study: the planar diver}

A planar diver (Crawford and Sastry, 1995) has the structure of a Lagrangian system in $\mathbb{R}^{3}$ with a cyclic coordinate (10), where the functions $a_{i 3}, i=1,2,3$, are highly nonlinear (trigonometric). The drift is caused by the constant angular momentum $\mu \neq 0$. A typical dive is a $1 \frac{1}{2}$ somersault pike. The steering problem is to lead the diver through the somersaults driven by the actuating arms and legs, and to enter the water in a fully extended vertical configuration. The time $T$ available to complete the dive is predetermined by gravity $g$, the initial vertical velocity $v_{0}$, and the distance $h$ the diver's center of mass must fall to reach the water:

$T=\frac{v_{0}+\sqrt{v_{0}^{2}+2 g h}}{g}$.

The configuration of the planar diver can be described by means of $\boldsymbol{\theta}=\left[\theta_{1}, \theta_{2}, \theta_{3}\right]^{\mathrm{T}}$, where $\theta_{1}$ is the angle between the legs and the trunk of the diver, $\theta_{2}$ is the angle between the arms and the trunk, and $\theta_{3}$ is the orientation of the trunk with respect to the vertical axis. Details can be found in (Crawford and Sastry, 1995). The motion planning problem for a dive is then to find a trajectory from a given initial state $\boldsymbol{\theta}^{0}$ to the desired final state $\boldsymbol{\theta}^{*}=[0, \pi,(2 k+1) \pi]^{\mathrm{T}}$, where $k \in \mathrm{Z}$ defines the number of somersaults in the dive.

In this system the drift term $\mu>0$ is good if the diver makes forward somersaults $(k>0)$. If however the diver with the same initial spin $\mu>0$ would want to make backward somersaults $(k<0)$, then the drift must be counteracted with heavy backward arm rotations in the opposite direction. In a real dive this is not possible due to physical constraints on the inputs. This is a good example of how the STLC property for driftless systems holds also with drift when the controls are sufficiently large by Lemma 2.1. However, if the constrained inputs are not sufficiently large for the diver, the system is not STLC at $\mathbf{x}^{0}$. Backward dives characterized by those $\mathbf{x}^{*}$ where $\theta_{3}^{*}<\theta_{3}^{0}$, cannot be carried out. This means that the initial point is not an interior point of the reachable set. The scheme discussed in this paper will work, however, since only forward dives are considered below. Time available is of course a concern that must be considered.

A symmetric diver is considered in order to simplify the presentation. In the general case the same procedure can be carried out with a little more tedious computation. More advanced dives can be approached in a similar manner.

The dynamic equations for a symmetric diver modified from Crawford and Sastry (1995) are

$\dot{\theta}_{1}=v_{1}$,

$\dot{\theta}_{2}=v_{2}$,

$\dot{\theta}_{3}=\frac{\mu}{a_{33}\left(\theta_{1}, \theta_{2}\right)}-\frac{a_{13}\left(\theta_{1}, \theta_{2}\right)}{a_{33}\left(\theta_{1}, \theta_{2}\right)} v_{1}-\frac{a_{23}\left(\theta_{1}, \theta_{2}\right)}{a_{33}\left(\theta_{1}, \theta_{2}\right)} v_{2}$,

where

$$
\begin{aligned}
a_{13}\left(\theta_{1}, \theta_{2}\right)= & {\left[\alpha+\beta \cos \left(\theta_{1}\right)+\zeta \cos \left(\theta_{2}-\theta_{1}\right)\right], } \\
a_{23}\left(\theta_{1}, \theta_{2}\right)= & {\left[\alpha+\beta \cos \left(\theta_{2}\right)+\zeta \cos \left(\theta_{2}-\theta_{1}\right)\right], } \\
a_{33}\left(\theta_{1}, \theta_{2}\right)= & \alpha_{3}+2 \beta\left[\cos \left(\theta_{1}\right)+\cos \left(\theta_{2}\right)\right] \\
& +2 \zeta \cos \left(\theta_{2}-\theta_{1}\right),
\end{aligned}
$$

where $\alpha, \beta, \zeta$, and $\alpha_{3}$ are physical constants given in Crawford and Sastry (1995).

Let $\mathbf{D}$ be a scaling matrix given by

$\mathbf{D}=\left[\begin{array}{cc}v_{1, \max } & 0 \\ 0 & v_{2, \max }\end{array}\right]$,

where $v_{1, \max }>0$ is the maximum angular velocity of the legs, and $v_{2, \max }>0$ is the maximum angular velocity of the arms. For simplicity let $v_{1, \max }=v_{2, \max }=A$.

The state and input transformations

$\mathbf{x}_{a}=\mathbf{D}^{-1}\left[\begin{array}{l}\theta_{1} \\ \theta_{2}\end{array}\right], \quad x_{3}=\theta_{3}, \quad \mathbf{u}=\mathbf{D}^{-1} \mathbf{v}$

give

$\dot{x}_{1}=u_{1}$,

$\dot{x}_{2}=u_{2}$,

$\dot{x}_{3}=\frac{\mu}{a_{33}\left(A x_{1}, A x_{2}\right)}-\frac{a_{13}\left(A x_{1}, A x_{2}\right)}{a_{33}\left(A x_{1}, A x_{2}\right)} A u_{1}$

$-\frac{a_{23}\left(A x_{1}, A x_{2}\right)}{a_{33}\left(A x_{1}, A x_{2}\right)} A u_{2}$, 
and the system defines vector fields $f, g_{1}$ and $g_{2}$ of the form (11) with scalars

$$
\begin{aligned}
& f=\frac{\mu}{a_{33}\left(A x_{1}, A x_{2}\right)}, \\
& g_{1}=-A \frac{a_{13}\left(A x_{1}, A x_{2}\right)}{a_{33}\left(A x_{1}, A x_{2}\right)}, \\
& g_{2}=-A \frac{a_{23}\left(A x_{1}, A x_{2}\right)}{a_{33}\left(A x_{1}, A x_{2}\right)} .
\end{aligned}
$$

\section{Path planning}

In this section an algorithm for open-loop path planning is derived for the class of systems presented in the previous section. The idea is to utilize the structure and to apply simple bang-bang controls in the planning.

Problem 4.1 (Path Planning Problem). Given an initial state $\mathbf{x}(0)=\mathbf{x}^{0}$ and a desired final state $\mathbf{x}^{*}$, find trajectories $\mathbf{x}(t) \in \mathscr{M}$ and inputs $\mathbf{u}(t) \in \mathscr{U}$, such that forward integration of

$\dot{\mathbf{x}}_{a}=\mathbf{u}$,

$\dot{x}_{n}=f\left(\mathbf{x}_{a}\right)+\sum_{i=1}^{n-1} g_{i}\left(\mathbf{x}_{a}\right) u_{i}$.

over the time interval $t \in[0, T]$, gives $\mathbf{x}(T)=\mathbf{x}^{*}$.

The final time $T$ may be free or given. Initial time is set to zero without loss of generality, since no vector field depends explicitly on time.

Consider piecewise bang-bang control $\mathbf{u}(t)$ with unitary bound

$\mathbf{u} \in \mathscr{U}=\left\{\mathbf{u} \in \mathbb{R}^{n-1}: u_{i} \in\{-1,0,1\}, \quad i \in\{1, \ldots, n-1\}\right\}$.

The amount of control available is a concern in the planning for this system due to the drift term. The class of bang-bang controls is often a sufficiently rich class of controls for analysis of nonlinear systems (Nijmeijer and van der Schaft, 1990). This simple class of controls makes it possible to integrate the equations forward in a very simple manner.

The following theorem by Sussmann (1983) will be helpful in the proof of convergence of the path planning procedure 4.1. The theorem below has been modified to fit $m$-input systems from the original version with $m=1$. The proof of the theorem is given by the same arguments as Sussmann (1983).

Theorem 4.1. Consider a real analytic system (1) with inputs constrained by $\left|u_{i}\right| \leq 1$. Suppose that for all $\mathbf{x} \in \mathscr{M}$ and for all integers $k>0$, there is a neighborhood $\mathscr{N} \subset \mathscr{M}$ of $\mathbf{x}$, where for all $\mathbf{x} \in \mathscr{N}$ and for all $i \in\{1, \ldots, m\}$, the term $\left[g_{i},(\operatorname{ad} \mathbf{f})^{k}\left(\mathbf{g}_{i}\right)\right](\mathbf{x})$ is a linear combination written $\sum_{j=0}^{k+1} \alpha_{j}(\operatorname{ad} \mathbf{f})^{j}\left(\mathbf{g}_{i}\right)(\mathbf{x})$ with real analytic coefficients $\alpha_{j}$ and $\left|\alpha_{k+1}\right|<1$. Then the real analytic system (1) has the property that whenever there exists a trajectory which connects two arbitrary points $\mathbf{x} \in \mathscr{N}$ in time $T$, then there exists a trajectory between the two same points in time $T$, which can be generated by a bang-bang control with a finite number of switches.

Lemma 4.1. The class of systems (21) considered here meets the sufficient conditions of Theorem 4.1 if there exist real analytic coefficients $\alpha_{0}$ and $\alpha_{1}$ such that for all $(i, j)$

$\frac{\partial^{2} f}{\partial x_{i} x_{j}}=\alpha_{0} f+\alpha_{1} \frac{\partial f}{\partial x_{i}}$.

This can be shown to be satisfied for the planar diver example.

The STLC property does not change if the discussion is restricted to piecewise constant controls, or even to bang-bang controls, under Assumption 2.1 (Sussmann, 1987). Hence it can be concluded that it is possible to steer the state $\mathbf{x}$ to $\mathbf{x}^{*}$ with bang-bang controls if the conditions of Lemma 4.1 are satisfied, since system (21) is STLC at $\mathbf{x}$ by Proposition 3.1.

Remark 4.1. The more general case where $u_{i} \in\left[-u_{i, \max }\right.$, $\left.u_{i, \max }\right]$ for some $u_{i, \max }>0$, is covered by the diffeomorphic input and state transformations

$\mathbf{u}^{\prime}=\mathbf{D}^{-1} \mathbf{u}, \quad \mathbf{x}_{a}{ }^{\prime}=\mathbf{D}^{-1} \mathbf{x}_{a}$,

where $\mathbf{D}=\operatorname{diag}\left(u_{1, \max }, \ldots, u_{n-1, \max }\right)$, so that the new inputs $u_{i}^{\prime} \in[-1,1]$. New scalars to consider are $f^{\prime}\left(\mathbf{x}_{a}^{\prime}\right)=f\left(\mathbf{D} \mathbf{x}_{a}^{\prime}\right)$, and $g_{i}^{\prime}\left(\mathbf{x}_{a}^{\prime}\right)=u_{i, \max } g_{i}\left(\mathbf{D} \mathbf{x}_{a}^{\prime}\right)$.

The time for steering the initial state $\mathbf{x}^{0}$ to the desired state $\mathbf{x}^{*}$ is partitioned into two parts as shown in Fig. 1. The control law in the first part will be termed as reaching

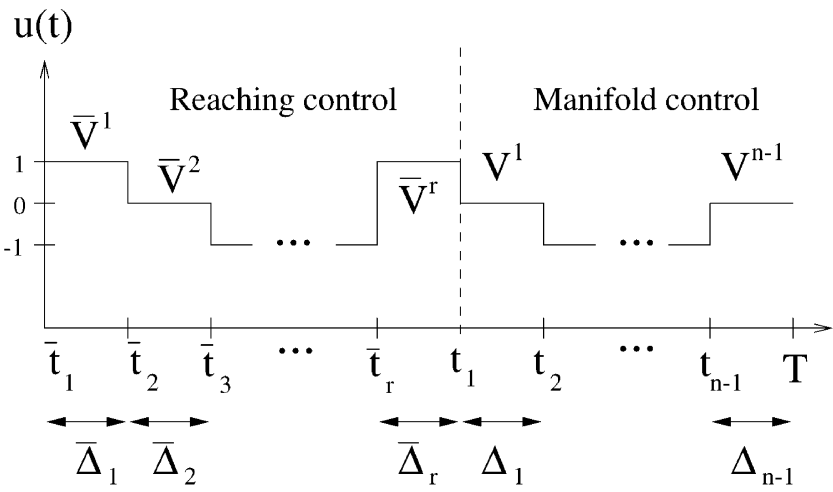

Fig. 1. Timing diagram of the sequence of control actions: reaching control for $t \in\left[\bar{t}_{1}, t_{1}\right)$ and manifold control for $t \in\left[t_{1}, T\right]$. 
control, since here the state is steered from the initial state to an $(n-1)$ dimensional manifold $\mathscr{S}_{V}$. The desidered behaviour of the system is to reach the desired state $\mathbf{x}^{*}$. Hence, the manifold $\mathscr{S}_{V}$ is designed so that $\mathbf{x}^{*}$ belongs to it and all the trajectories contained in it converge to $\mathbf{x}^{*}$. The control of the system on the manifold will be called manifold control.

\subsection{Reaching control}

Let the reaching control be defined by a sequence of $r \geq 1$ piecewise constant signals as follows:

$u_{k}(t)=\bar{v}_{k, i}, \quad t \in\left[\bar{t}_{i}, \bar{t}_{i+1}\right), i \in\{1, \ldots, r\}$

for all $k \in\{1, \ldots, n-1\}$, where $\bar{v}_{k, i} \in\{-1,0,1\}, r \geq 1$ is the number of control switches in the reaching control segment, $\bar{t}_{1}=0$ is the starting time, $\bar{t}_{r+1}$ is the time where the reaching control ends and the manifold control starts. Let

$\bar{\Delta}_{i}=\bar{t}_{i+1}-\bar{t}_{i} \geq 0, \quad \bar{\Delta}=\left[\bar{\Delta}_{1}, \ldots, \bar{\Delta}_{r}\right]^{\mathrm{T}}$.

Define the $(n-1) \times r$ reaching control matrix $\overline{\mathbf{V}}$ by

$\overline{\mathbf{V}}=\left[\begin{array}{ccc}\bar{v}_{1,1} & \cdots & \bar{v}_{1, r} \\ \vdots & \ddots & \vdots \\ \bar{v}_{n-1,1} & \cdots & \bar{v}_{n-1, r}\end{array}\right]$

so that the control $\mathbf{u}$ during the time interval $\mathbf{t} \in\left[\bar{t}_{i}, \bar{t}_{i+1}\right]$ is given by the $i$ th column $\overline{\mathbf{V}}^{i}$ of $\overline{\mathbf{V}}$.

Forward integration of system (21) with initial state $\mathbf{x}^{0}$ gives $\mathbf{x}\left(t_{1}\right)=\mathbf{x}^{1}$ at time $t_{1}=\bar{t}_{r+1}=\sum_{i=1}^{r} \bar{\Delta}_{i}$,

$\mathbf{x}_{a}^{1}=\mathbf{x}_{a}^{0}+\overline{\mathbf{V}} \bar{\Delta}$

$$
\begin{aligned}
x_{n}^{1}= & x_{n}^{0}+\sum_{i=1}^{r} \int_{0}^{\bar{\Delta}_{i}} f\left(\mathbf{x}_{a}^{0}+\overline{\mathbf{V}} \mathbf{Z}^{i-1} \overline{\boldsymbol{\Delta}}+\overline{\mathbf{V}}^{i} \tau\right) \mathrm{d} \tau \\
& +\sum_{i=1}^{r} \sum_{j=1}^{n-1} \bar{v}_{j, i} \int_{0}^{\overline{\boldsymbol{\Delta}}_{i}} g_{j}\left(\mathbf{x}_{a}^{0}+\overline{\mathbf{V}} \mathbf{Z}^{i-1} \overline{\boldsymbol{\Delta}}+\overline{\mathbf{V}}^{i} \tau\right) \mathrm{d} \tau,
\end{aligned}
$$

where $\mathbf{Z}^{i}$ is the $r \times r$ matrix whose first $i$ columns are the first $i$ columns of the $r \times r$ identity matrix while the others are zero columns.

\subsection{Manifold control}

Let the manifold controls be given by the $(n-1)$ piecewise constant signals

$u_{k}(t)=v_{k, i}, \quad t \in\left[t_{i}, t_{i+1}\right), i \in\{1, \ldots, n-1\}$

for all $k \in\{1, \ldots, n-1\}$, where $v_{k, i} \in\{-1,0,1\}$, and $t_{n}=T$ is the time available. Let

$\Delta_{i}=t_{i+1}-t_{i} \geq 0, \quad \Delta=\left[\Delta_{1}, \ldots, \Delta_{n-1}\right]^{\mathrm{T}}$
Define the $(n-1) \times(n-1)$ manifold control matrix $\mathbf{V}$ by

$\mathbf{V}=\left[\begin{array}{ccc}v_{1,1} & \cdots & v_{1, n-1} \\ \vdots & \ddots & \vdots \\ v_{n-1,1} & \cdots & v_{n-1, n-1}\end{array}\right]$

so that the control $\mathbf{u}$ during the time interval $t \in\left[t_{i}, t_{i+1}\right]$ is given by the $i$ th column $\mathbf{V}^{i}$ of $\mathbf{V}$.

Imposing $\mathbf{x}(T)=\mathbf{x}^{*}$, forward integration of system (21) with intial state $\mathbf{x}^{1}$, at final time $T=t_{1}+\sum_{i=1}^{n-1} \Delta_{i}$, gives

$$
\begin{aligned}
\mathbf{x}_{a}^{*}= & \mathbf{x}_{a}^{1}+\mathbf{V} \boldsymbol{\Delta}, \\
x_{n}^{*}= & x_{n}^{1}+\sum_{i=1}^{n-1} \int_{0}^{\boldsymbol{\Delta}_{i}} f\left(\mathbf{x}_{a}^{1}+\mathbf{V} \mathbf{Z}^{i-1} \boldsymbol{\Delta}+\mathbf{V}^{i} \tau\right) \mathrm{d} \tau \\
& +\sum_{i=1}^{n-1} \sum_{j=1}^{n-1} v_{j, i} \int_{0}^{\boldsymbol{\Delta}_{i}} g_{j}\left(\mathbf{x}_{a}^{1}+\mathbf{V} \mathbf{Z}^{i-1} \boldsymbol{\Delta}+\mathbf{V}^{i} \tau\right) \mathrm{d} \tau .
\end{aligned}
$$

Define an $(n-1)$ dimensional manifold $\mathscr{S}_{V}$ by

$\mathscr{S}_{V}=\left\{\mathbf{x} \in \mathscr{M} \mid \sigma_{V}(\mathbf{x})=0\right\}$,

where

$$
\begin{aligned}
\sigma_{V}(\mathbf{x})= & x_{n}-x_{n}^{*}+\sum_{i=1}^{n-1} \int_{0}^{\Delta_{i}} f\left(\mathbf{x}_{a}+\mathbf{V} \mathbf{Z}^{i-1} \Delta+\mathbf{V}^{i} \tau\right) \mathrm{d} \tau \\
& +\sum_{i=1}^{n-1} \sum_{j=1}^{n-1} v_{j, i} \int_{0}^{\boldsymbol{\Delta}_{i}} g_{j}\left(\mathbf{x}_{a}+\mathbf{V} \mathbf{Z}^{i-1} \boldsymbol{\Delta}+\mathbf{V}^{i} \tau\right) \mathrm{d} \tau .
\end{aligned}
$$

Note that $\sigma_{V}(\mathbf{x})$ only is defined for those $\mathbf{x}$ where all entries $\Delta_{i}$ in

$\Delta=\mathbf{V}^{-1}\left(\mathbf{x}_{a}^{*}-\mathbf{x}_{a}\right)$

are nonnegative.

$\mathbf{V}$ can always be selected so that $\boldsymbol{\Delta}_{i} \geq 0$ in Eq. (35) and $\operatorname{det}(\mathbf{V}) \neq 0$. This can be obtained by $\mathbf{V}=$ $\operatorname{diag}\left(\operatorname{sgn}\left(x_{1}^{*}-x_{1}\right), \ldots, \operatorname{sgn}\left(x_{n-1}^{*}-x_{n-1}\right)\right)$, so that $\Delta_{i}=$ $\left|x_{i}^{*}-x_{i}\right| \geq 0$ for $i \in\{1, \ldots, n-1\}$.

With definition (33) of $\mathscr{S}_{V}$, the following is given by design.

Proposition 4.1. Any state $\mathbf{x} \in \mathscr{S}_{V}$ can be steered to the desired state $\mathbf{x}^{*}$ by a sequence of switching controls (29), so that $\mathbf{x}\left(t_{1}\right)=\mathbf{x}^{1} \in \mathscr{S}_{V} \Rightarrow \mathbf{x}(T)=\mathbf{x}^{*}$.

By inserting Eq. (28) into Eq. (34) and setting $\sigma_{V}=0$, the result is

$$
\begin{aligned}
x_{n}^{*}- & x_{n}^{0} \\
= & \sum_{i=1}^{r} \int_{0}^{\bar{\Delta}_{i}} f\left(\mathbf{x}_{a}^{0}+\overline{\mathbf{V}} \mathbf{Z}^{i-1} \overline{\boldsymbol{\Delta}}+\overline{\mathbf{V}}^{i} \tau\right) \mathrm{d} \tau \\
& +\sum_{i=1}^{r} \sum_{j=1}^{n-1} \bar{v}_{j, i} \int_{0}^{\bar{\Delta}_{i}} g_{j}\left(\mathbf{x}_{a}^{0}+\overline{\mathbf{V}} \mathbf{Z}^{i-1} \bar{\Delta}+\overline{\mathbf{V}}^{i} \tau\right) \mathrm{d} \tau \\
& +\sum_{i=1}^{n-1} \int_{0}^{\Delta_{i}} f\left(\mathbf{x}_{a}^{0}+\overline{\mathbf{V}} \overline{\boldsymbol{\Delta}}+\mathbf{V} \mathbf{Z}^{i-1} \boldsymbol{\Delta}+\mathbf{V}^{i} \tau\right) \mathrm{d} \tau
\end{aligned}
$$




$$
\begin{aligned}
& +\sum_{i=1}^{n-1} \sum_{j=1}^{n-1} v_{j, i} \int_{0}^{\Delta_{i}} g_{j}\left(\mathbf{x}_{a}^{0}+\overline{\mathbf{V}} \bar{\Delta}+\mathbf{V} \mathbf{Z}^{i-1} \Delta+\mathbf{V}^{i} \tau\right) \mathrm{d} \tau \\
= & \Psi_{\mathbf{V}, \overline{\mathbf{V}}}\left(\overline{\boldsymbol{\Delta}}, \mathbf{x}_{a}^{0}\right),
\end{aligned}
$$

where $\boldsymbol{\Delta}$ is given by

$\Delta=\mathbf{V}^{-1}\left(\mathbf{x}_{a}^{*}-\mathbf{x}_{a}^{0}-\overline{\mathbf{V}} \overline{\mathbf{\Delta}}\right)$.

Eq. (36) can be solved with respect to a $\bar{\Delta}$ with nonnegative entries for a given pair $\left(\mathbf{x}^{0}, \mathbf{x}^{*}\right)$ by Newton's method (Luenberger, 1984). That is, there exists a set of matrices $(\mathbf{V}, \overline{\mathbf{V}})$ that gives nonnegative entries in $\overline{\boldsymbol{\Delta}}$. Thereafter, $\boldsymbol{\Delta}$ is found from Eq. (37).

From this it can be concluded that the control matrix (27) will steer the state $\mathbf{x}$ from the initial $\mathbf{x}^{0}$ to the manifold $\mathscr{S}_{V}$ if $x_{n}^{*} \in\left[x_{n}^{0}+\Psi_{\mathbf{V}, \mathbf{V}}^{\min }, x_{n}^{0}+\Psi_{\mathbf{V}, \mathbf{V}}^{\max }\right]$. These bounds can be obtained by solving the optimization problems

$\Psi_{\mathbf{V}, \mathbf{V}}^{\min }= \begin{cases}\text { minimize } & \Psi_{\mathbf{V}, \overline{\mathbf{v}}}\left(\overline{\boldsymbol{\Delta}}, \mathbf{x}_{a}^{0}\right) \\ \text { subject to all } & \overline{\boldsymbol{\Delta}}_{i} \geq 0,\end{cases}$
$\Psi_{\mathbf{V}, \mathbf{V}}^{\max }= \begin{cases}\text { maximize } & \Psi_{\mathbf{V}, \overline{\mathbf{v}}}\left(\overline{\boldsymbol{\Delta}}, \mathbf{x}_{a}^{0}\right) \\ \text { subject to all } & \overline{\boldsymbol{\Delta}}_{i} \geq 0 .\end{cases}$

Methods for solving Eq. (38) can be found in Luenberger (1984). Note that the result of the optimization might be infinite.

The result of this analysis can be summarized as follows:

Proposition 4.2. Any $x_{n}$ in the interval

$\mathscr{I}=\left[x_{n}^{0}+\Psi_{\mathbf{V}, \mathbf{V}}^{\min }, \mathbf{x}_{n}^{0}+\Psi_{\mathbf{V}, \mathbf{V}}^{\max }\right]$

can be reached with the reaching control $\overline{\mathbf{V}}$ (27) and the manifold control $\mathbf{V}$ (31). Further it can be concluded by Proposition (4.1) that if time is unconstrained and the system is $S T L C$ everywhere, then the union of $\mathscr{I}$ for all pairs $(\mathbf{V}, \overline{\mathbf{V}})$ is the whole $\mathbb{R}$.

\subsection{Path planning procedure}

Procedure 4.1 is derived from the discussion above which provides solutions for the path planning problem for the pair of an initial state $\mathbf{x}^{0}$ and a desired final state $\mathbf{x}^{*}$.

\section{Procedure 4.1}

- Initialization.

Initialize $r=1$.

Enumerate all possible nonsingular

$(n-1) \times(n-1)$ matrices $V$ of

type (31) by $V_{k}, 1 \leq k \leq k_{\max }$,

where $k_{\max } \leq 3^{(\mathrm{n}-1)^{2}}$.

Also enumerate all possible

$(n-1) \times r$ matrices $\overline{\mathbf{v}}$ of type (27)

by $\overline{\mathbf{v}}_{1}$, where $1 \leq 1 \leq l_{\max } \leq 3^{(n-1) r}$

Initialize $k=1=1$.

- Step 1

Optimize (38) and establish the reachable set

$\mathscr{S}^{k, 1}=\left[X_{n}^{0}+\Psi_{\mathbf{v}_{k}, \mathbf{v}_{k}}^{\min }, X_{n}^{0}+\Psi_{\mathbf{V}_{k}, \mathbf{v}_{k}}^{\min }\right]$

with controls $\mathbf{V}^{*}$ and $\mathbf{V}_{\mathrm{k}}$.

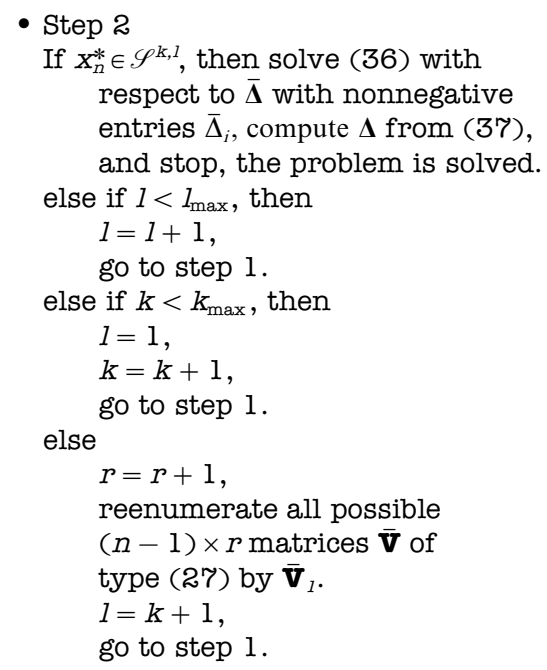

From the discussion above the following can be concluded.

Proposition 4.3. Procedure 4.1 solves Problem 4.1 and converges if the controls are sufficiently large, the system is STLC from any $\mathbf{x} \in \mathscr{M}$, and satisfies the condition of Lemma 4.1.

Remark 4.2. A more flexible procedure regarding the order of investigation of the different pairs $\left(\mathbf{V}_{k}, \overline{\mathbf{V}}_{l}\right)$ would improve the efficiency of Procedure 4.1. The next pair $\left(\mathbf{V}_{k}, \overline{\mathbf{V}}_{l}\right)$ should be selected in view of the results of the procedure so far.

A slight modification of Procedure 4.1 allows a computation of a subset of the reachable set. This set is restricted when time $T$ available is restricted by some physical constraint, for example. This procedure can be found in Godhavn (1997).

\subsection{Case study: path planning for the planar diver}

Using procedure 4.1, a $\mathbf{V}$ can be found as

$\mathbf{V}=\left[\begin{array}{cc}\operatorname{sgn}\left(x_{1}^{*}-x_{1}\right) & 0 \\ 0 & \operatorname{sgn}\left(x_{2}^{*}-x_{2}\right)\end{array}\right]$

with the nice property $\mathbf{V}^{-1}=\mathbf{V}$. This $\mathbf{V}$ gives the following $\Delta$ :

$\boldsymbol{\Delta}=\left[\begin{array}{l}\left|x_{1}^{*}-x_{1}\right| \\ \left|x_{2}^{*}-x_{2}\right|\end{array}\right]$.

With Eq. (40), $\theta_{1}$ is controlled first, and $\theta_{2}$ last. Integration of Eq. (16) as in Eq. (34) gives

$$
\begin{aligned}
\sigma_{V}(x)= & A\left(x_{3}-x_{3}^{*}-\frac{1}{2}\left(x_{1}^{*}-x_{1}\right)-\frac{1}{2}\left(x_{2}^{*}-x_{2}\right)\right) \\
& +\left(a\left(A x_{2}\right)+\frac{b\left(A x_{2}\right)}{A \operatorname{sgn}\left(x_{1}^{*}-x_{1}\right)}\right)
\end{aligned}
$$




$$
\begin{aligned}
& \times\left(f_{c\left(A x_{2}\right)}\left(A x_{1}^{*}-\phi\left(A x_{2}\right)\right)\right. \\
& \left.-f_{c\left(A x_{2}\right)}\left(A x_{1}-\phi\left(A x_{2}\right)\right)\right) \\
& +\left(a\left(A x_{1}^{*}\right)+\frac{b\left(A x_{1}^{*}\right)}{A \operatorname{sgn}\left(x_{2}^{*}-x_{2}\right)}\right) \\
& \times\left(f_{c\left(A x_{1}^{*}\right)}\left(A x_{2}^{*}-\phi\left(A x_{1}^{*}\right)\right)\right. \\
& \left.-f_{c\left(A x_{2}^{*}\right)}\left(A x_{2}-\phi\left(A x_{1}^{*}\right)\right)\right)
\end{aligned}
$$

where

$f_{c}(x)=\arctan \left(c \tan \left(\frac{x}{2}\right)\right)+\pi k(x) \quad$ with $c \in \mathbb{R}$

and $k: \mathbb{R} \mapsto \mathrm{Z}$ is a piecewise constant mapping, such that $f_{c}(\cdot)$ is continuous over and $f_{c}(0)=0$. The functions $a(s)$, $b(s), c(s), \phi(s)$ and $\psi(s)$ are given by

$\phi(s)=\arctan 2(\zeta \sin (s), \beta+\zeta \cos (s))$,

$\psi(s)=\sqrt{\zeta^{2}+\beta^{2}+2 \zeta \beta \cos (s)}$

$a(s)=\frac{\alpha_{3}+2 \beta \cos (s)-2 \alpha}{\sqrt{\left(\alpha_{3}+2 \beta \cos (s)\right)^{2}-4 \psi(s)^{2}}}$,

$b(s)=\frac{2 \mu}{\sqrt{\left(\alpha_{3}+2 \beta \cos (s)\right)^{2}-4 \psi(s)^{2}}}$,

$c(s)=\frac{\sqrt{\left(\alpha_{3}+2 \beta \cos (s)\right)^{2}-4 \psi(s)^{2}}}{\alpha_{3}+2 \beta \cos (s)+2 \psi(s)}$

with parameters $A=10, \mu=70, \alpha_{3}=11.234, \beta=2.299$, $\alpha=3.732$, and $\zeta=-0.207$.

First the effect of the drift on the system can be seen by letting $\overline{\mathbf{V}}$ be given by

$\overline{\mathbf{V}}=\left[\begin{array}{l}0 \\ 0\end{array}\right]$.

From Eq. (16) and with zero controls it is obtained

$x_{1}^{1}=x_{1}^{0}$,

$x_{2}^{1}=x_{2}^{0}$,

$x_{3}^{1}=x_{3}^{0}+\frac{\mu}{A a_{33}\left(A x_{1}^{0}, A x_{2}^{0}\right)} \bar{\Delta}$.

With this initial zero control interval, all initial states $\mathbf{x}^{0}$ in a set $\mathscr{2}_{I}^{0} \subset \mathbb{R}^{3}$ given by

$$
\begin{aligned}
\mathscr{Q}_{I}^{0} & =\left\{\mathbf{x} \in \mathscr{M} \mid \sigma_{V}\left(x_{1}, x_{2}, x_{3}+\frac{\mu}{A a_{33}\left(A x_{1}, A x_{2}\right)} \bar{\Delta}\right)\right. \\
& \left.=0,0 \leq \bar{\Delta} \leq \bar{\Delta}_{\max }\right\}
\end{aligned}
$$

for some given $\bar{\Delta}_{\max }$, can be steered so that $\mathbf{x}(T)=\mathbf{x}^{*}$.

The set $\mathscr{Q}_{I}^{0}$ with $0 \leq \bar{\Delta} \leq 1$ is shown in Fig. 2 . All states between the top surface $\mathscr{S}_{V}$ and the bottom surface with $\bar{\Delta}=1$ can reach the desired state with control (40) and (43).

The effect of applying another reaching control can be seen by selecting the following $\overline{\mathbf{V}}$ :

$\overline{\mathbf{V}}=\left[\begin{array}{c}0 \\ -1\end{array}\right]$

which results in

$x_{1}^{1}=x_{1}^{0}$,

$x_{2}^{1}=x_{2}^{0}-\bar{\Delta}$,

$$
\begin{aligned}
x_{3}^{1}= & x_{3}^{0}+\frac{A \bar{\Delta}}{2}+\left(a\left(A x_{1}^{0}\right)-\frac{b\left(A x_{1}^{0}\right)}{A}\right) \\
& \times\left(f_{c\left(A x_{2}^{0}\right)}\left(A x_{2}^{0}-A \bar{\Delta}-\phi\left(A x_{1}^{0}\right)\right)\right. \\
& \left.-f_{c\left(A x_{2}^{0}\right)}\left(A x_{2}^{0}-\phi\left(A x_{1}^{0}\right)\right)\right) .
\end{aligned}
$$

With this initial reaching control applied in a time interval $[0, \bar{\Delta}]$, all initial states $\mathbf{x}^{0}$ in a set $\mathscr{Q}_{I}^{1} \subset \mathbb{R}^{3}$ defined by

$\mathscr{Q}_{I}^{1}=\left\{\mathbf{x} \in \mathscr{M} \mid \sigma_{V}\left(\mathbf{x}^{1}\right)=0,0 \leq \bar{\Delta} \leq \bar{\Delta}_{\max }\right\}$

with $\mathbf{x}^{1}=\left[x_{1}^{1}, x_{2}^{1}, x_{3}^{1}\right]^{\mathrm{T}}$ as in Eq. (47), and for some $\bar{\Delta}_{\max }$, can be steered so that $\mathbf{x}(T)=\mathbf{x}^{*}$.

The set $\mathscr{Q}_{I}^{1}$ with $0 \leq \bar{\Delta} \leq 1$ is shown in Fig. 3. All states between the top surface $\mathscr{S}_{V}$ and the bottom surface $(\bar{\Delta}=1)$ can reach the desired state with the control matrices above.

A realistic initial condition to use for the diver model for a $1 \frac{1}{2}$ somersault dive is $\boldsymbol{\theta}=[0, \pi, 0]^{\mathrm{T}}$ and a desired final state $\boldsymbol{\theta}^{*}=[0, \pi, 3 \pi]^{\mathrm{T}}$. In order to generate a motion similar to the one of a real diver, this control sequence is applied:

$$
\overline{\mathbf{V}}=\left[\begin{array}{ccc}
0 & 1 & 0 \\
-1 & 0 & 0
\end{array}\right], \quad \mathbf{V}=\left[\begin{array}{cc}
-1 & 0 \\
0 & 1
\end{array}\right]
$$

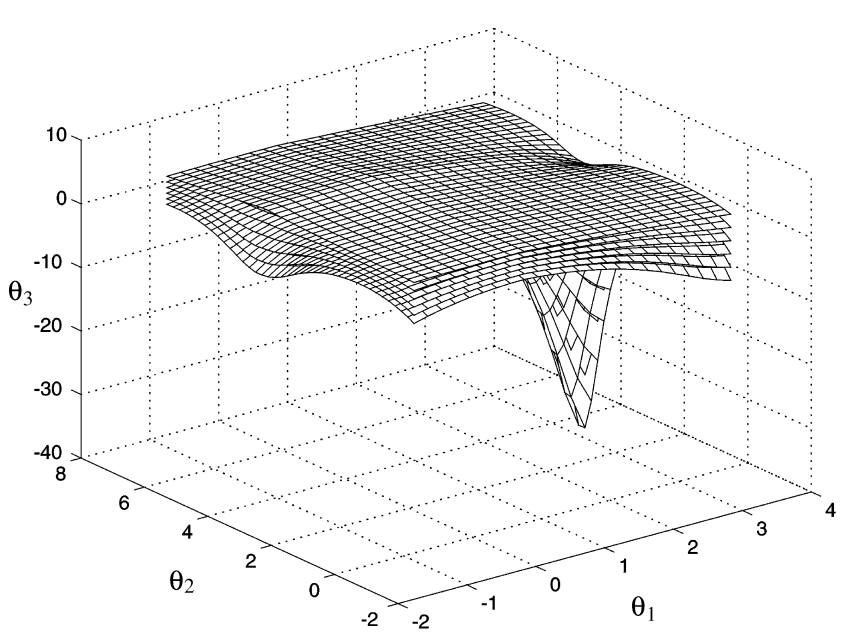

Fig. 2. The set $\mathscr{Q}_{I}^{0}$ for the diver example with $0 \leq \bar{\Delta} \leq 1$. The top surface is the original manifold $\mathscr{S}_{V}$, which corresponds to $\bar{\Delta}=0$. 


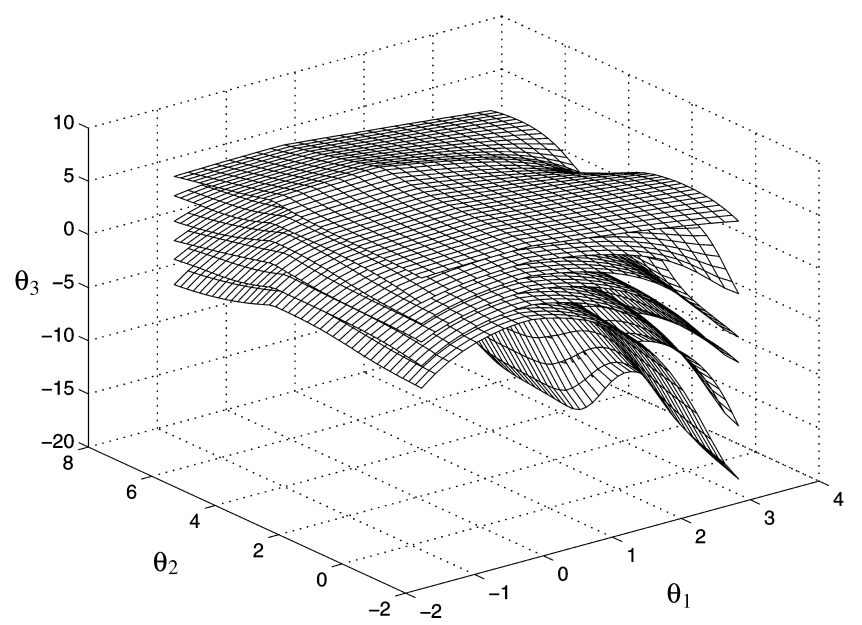

Fig. 3. The set $\mathscr{2}_{I}^{1}$ for the diver example with $0 \leq \bar{\Delta} \leq 1$. The top surface is the original manifold $\mathscr{S}_{V}$, which corresponds to $\bar{\Delta}=0$.

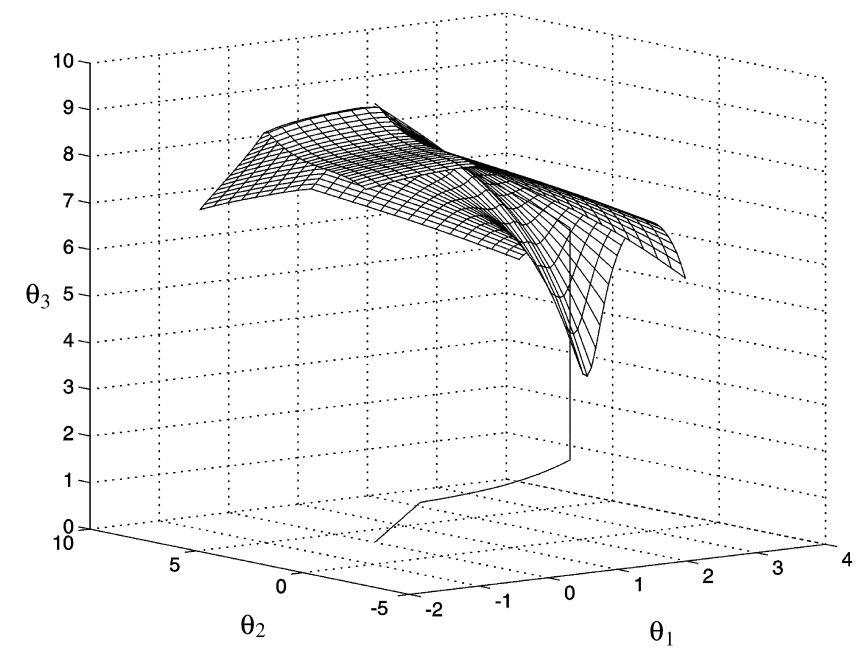

Fig. 4. Evolution of the diver state. Initially the state follows the line shown with reaching control until it hits the manifold $\mathscr{S}_{V}$. After that the trajectory stays on $\mathscr{S}_{V}$ until the desired state is reached.
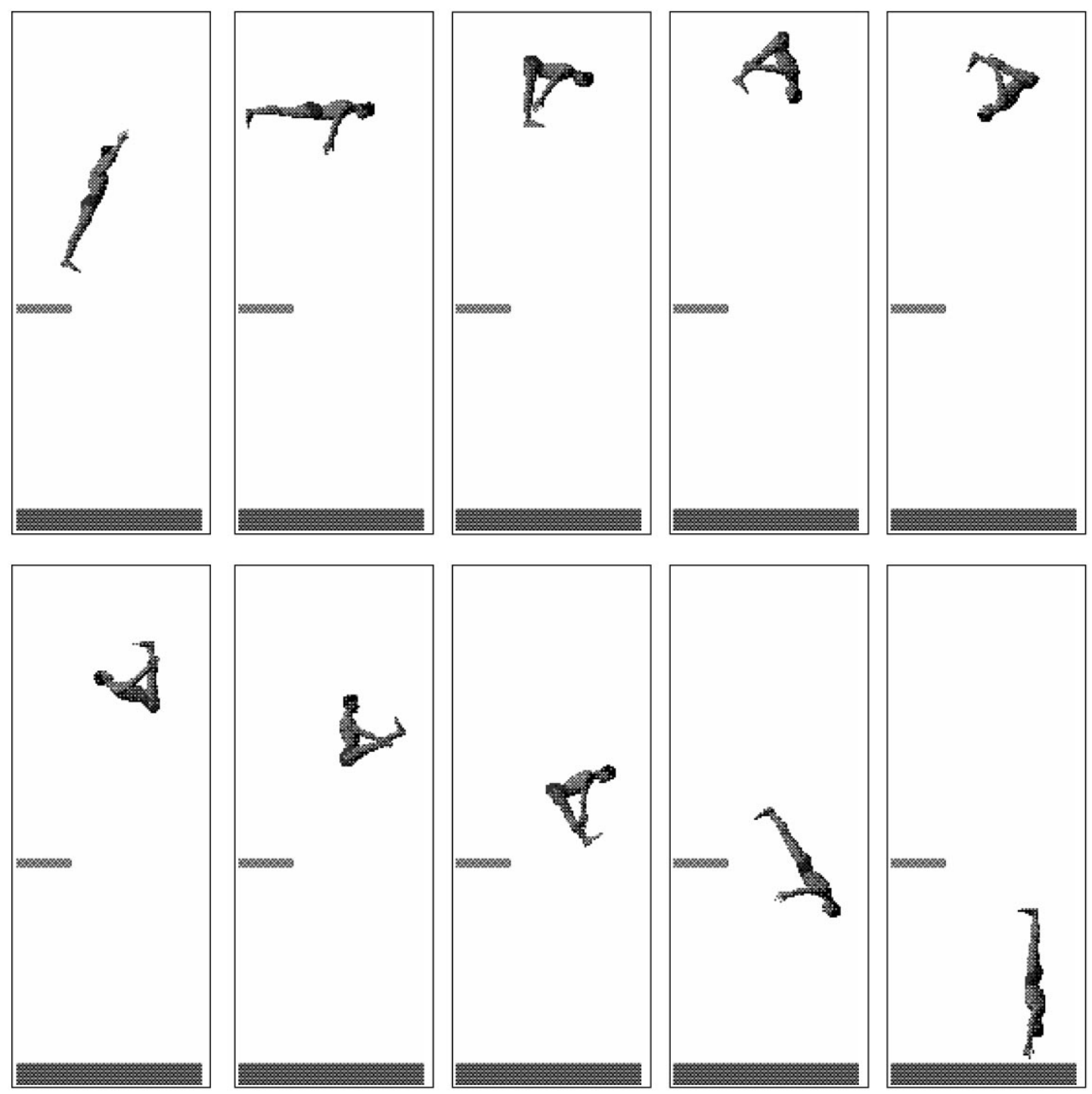

Fig. 5. Frames from an animation of the simulation results with a symmetric model of a planar diver and bang-bang controls. 
The predetermined time $T$ available to complete the dive puts an additional constraint on the choice of control intervals:

$$
\sum_{i=1}^{3} \bar{\Delta}_{i}+\sum_{i=1}^{2} \Delta_{i}=T .
$$

This time constraint defines a subset of the manifold $\mathscr{S}_{V}$. The number of free variables decreases by one due to this constraint, and hence it is necessary to have $r \geq 2$. Free variables are $\bar{\Delta}_{i} \geq 0$ with $i \in\{1, \ldots, r\}$.

Using the equations presented above, an appropriate choice of control intervals was found to steer the diver from the initial to the final configuration in $1.7 \mathrm{~s}$. The trajectory is shown in Figs. 4 and 5.

\section{Conclusion and future work}

The control problem for a class of nonholonomic systems with drift has been studied. A lemma for controllability for systems with nonvanishing drift was presented. The path planning problem was solved with a procedure providing numeric solutions with bangbang controls, and the procedure is guarenteed to solve the problem under a necessary controllability assumption. This procedure was applied to the mathematical model of a planar diver.

Future work should concentrate on extending the results to larger classes of controls, including smooth inputs, and to close the loop by feedback control.

\section{Acknowledgements}

The paper has been partially supported by NATO under grant CRG 960750.

\section{References}

Aeyels, D. (1984). Local and global controllability for nonlinear systems. Systems and Control Letters, 5, 19-26.

Arnold, V. I. (1989). Mathematical methods of classical mechanics (2nd ed.). New York: Springer.

Bartels, R. H., Beatty, J. C., \& Barsky, B. A. (1987). An introduction to splines for use in computer graphics and geometric modeling. Los Altos, CA: Morgan Kaufmann.

Bloch, A. M., Reyhanoglu, M., \& McClamroch, N. H. (1992). Control and stabilization of nonholonomic dynamic systems. IEEE Trans. Automat. Control, 37(11), 1746-1753.

Brockett, R. W. (1983). Asymptotic stability and feedback stabilization. Differential geometric control theory (pp. 181-191). Boston: Birkhauser.

Crawford, L. S., \& Sastry, S. S. (1995). Biological motor control approaches for a planar diver. Proc. from the 34th IEEE Conf. on Decision and Control (pp. 3881-3886). New Orleans, LA.

Di Giamberardino, P., Monaco, S., \& Normand-Cyrot, D. (1996). Digital control through finite feedback discretizability. Proc. IEEE Conf. on Robotics and Automation. pp. 3191-3146. Minneapolis.

Godhavn, J.-M. (1997). Topics in nonlinear motion control: non-holonomic, underactuated, and hybrid systems. Ph.D. Thesis. NTNU, Norway.
Goldstein, H. (1980). Classical mechanics (2nd ed.). Reading, MA: Addison-Wesley.

Hermes, H., Lundell, A., \& Sullivan, D. (1984). Nilpotent bases for distributions and control systems. J. Differential Equations, 55, 385-400.

Kailath, T. (1980). Linear systems. Englewood Cliffs, NJ: Prentice-Hall.

Kapitanovsky, A., Goldenberg, A. A., \& Mills, J. K. (1993). Dynamic control and motion planning technique for a class of nonlinear systems with drift. Systems Control Lett., 21, 363-369.

Kawski, M. (1988). Nilpotent lie algebras of vectorfields. J. fur Die Reine Angewandte Math., 388, 1-17.

Kawski, M. (1993). Combinatorics of realizations of nilpotent control systems. In: M. Fliess (Ed.), Nonlinear Control Systems Design, Selected papers of IF AC symposium, (pp. 639-644).

Kelly, S. D., \& Murray, R. M. (1996). The geometry and control of dissipative systems. Proc. 35th IEEE Conf. on Decision and Control. pp. 981-986. Kobe, Japan.

Kolmanovsky, I. (1995). On motion planning and feedback control of nonholonomic dynamic systems with applications to attitude control of multibody systems. Ph.D. Thesis. University of Michigan, Ann Arbor.

Kolmanovsky, I., \& McClamroch, N. H. (1995). Application of integrator backstepping to nonholonomic control problems. Preprints of Nonlinear Control Systems Design Symp.

Kreyszig, E. (1988). Advanced engineering mathematics. (6th ed.) New York: Wiley.

Lewis, A. D., \& Murray, R. M. (1997). Configuration controllability of simple mechanical control systems. SIAM J. Control Optim. 35(3), pp 766-790 also CDS Technical Report 95-015.

Luenberger, D. G. (1984). Linear and nonlinear programming (2nd ed.). Reading MA: Addison-Wesley.

Martin, P. (1993). A geometric sufficient condition for flatness of systems with $m$ inputs and $m+1$ states. Proc. 32nd IEEE Conf. on Decision and Control (pp. 3431-3436). San Antonio, Texas.

M'Closkey, R. T., \& Murray, R. M. (1994). Extending exponential stabilizers for nonholonomic systems from kinematic controllers to dynamic controllers. Preprints of the 4th IFAC Symp. on Robot Control. Capri, Italy.

Murray, R. M. (1994). Nilpotent bases for a class of nonintegrable distributions with applications to trajectory generation for nonholonomic systems. Math. Control Signals Systems, 7(1), 58-75.

Murray, R. M., \& Sastry, S. S. (1993). Nonholonomic motion planning: steering using sinusoids. IEEE Trans. Automat. Control, 38(5), 700-716.

Nijmeijer, H., \& van der Schaft, A. J. (1990). Nonlinear dynamical control systems. New York: Springer.

Pomet, J.-B., \& Samson, C. (1993). Time-varying exponential stabilization of nonholonomic systems in power form. Technical report. 2126, INRIA, France.

Rathinam, M., \& Murray, R. M. (1996). Configuration flatness of Lagrangian systems underactuated by one control. Proc. 35th IEEE Conf. on Decision and Control. pp. 1688-1693. Kobe, Japan.

Rouchon, P., Fliess, M., Levine, J., \& Martin, P. (1993). Flatness, motion planning and trailer systems. Proc. 32nd IEEE Conf. on Decision and Control. (pp. 2700-2705). San Antonio, Texas.

Sussmann, H. J. (1983). Lie brackets, real analyticity and geometric control. In R. W. Brockett, R. S. Millman, \& H. J. Sussmann (Eds.), Differential geometric control theory of Progress in Mathematics. Vol. 27 (pp. 1-117). Boston: Birkhauser

Sussmann, H. J. (1987). A general theorem on local controllability. SIAM J. Control Optim. 25(1), 158-194.

Teel, A. R., Murray, R. M., \& Walsh, G. (1992). Nonholonomic control systems: from steering to stabilization with sinusoids. Proc. IEEE Control and Decision Conf.

Tilbury, D., \& Sastry, S. (1995). The multi-steering N-trailer system: a case study of Goursat normal forms and prolongations. Int. J. Robust Nonlinear Control, 5(4), 343-364. 
Tilbury, D., Murray, R. M., \& Sastry, S. S. (1995). Trajectory generation for the $\mathrm{N}$-trailer problem using Goursat normal form. IEEE Trans. Automat. Control, 40(5), 802-819.

Varaiya, P. (1993). Smart cars on smart roads: problems of control. IEEE Trans. Automat. Control, 38(2), 195-207.

Walsh, G. C., Sarti, A., \& Sastry, S. S. (1993). Algorithms for steering on the group of rotations. Proc. Amer. Control Conf. San Francisco, California.

Walsh, G. C., \& Sastry, S. S. (1991). On reorienting rigid linked bodies using internal motions. Proc. 30th IEEE Conf. on Decision and Control (pp. 1190-1195). Brighton, England.

Walsh, G., Tilbury, D., Sastry, S., Murray, R., \& Laumond, J. P. (1994). Stabilization for systems with nonholonomic constraints. IEEE Trans. Automat. Control, 39(1), 216-222.

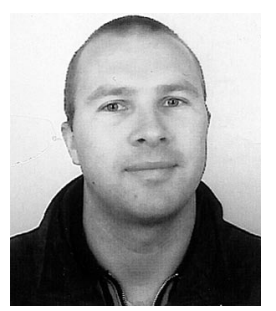

John-Morten Godhavn received the sivilingeniør (M.Sc.) degree from the Department of Engineering Cybernetics at the Norwegian Institute of Technology $(\mathrm{NTH})$ in 1992 and the doktor ingeniør (Ph.D.) from the Department of Engineering Cybernetics at the Norwegian University of Science and Technology (NTNU) in 1997. He was a visting scholar at the Department of Electrical Engineering and Computer Science, University of California at Berkeley, in 1995 and 1996. Currently he is working as a project engineer at Seatex AS in Trondheim, Norway. His research interests include nonlinear control systems, control of ocean vehicles, hybrid systems, and navigation systems.

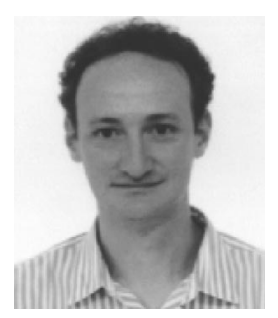

Andrea Balluchi received, from the University of Pisa, the Laurea degree (summa cum laude) in Electrical Engineering in 1993, and the Ph.D. in Automation and Industrial Robotics in 1997. He was a visiting scholar at the Department of Computer Science of the University of Sheffield, in 1992; at the Laboratoire d'Analyse et d'Architecture des Systemes CNRS in Toulouse, in 1995; and at the Department of Electrical Engineering and Computer Science of the University of California at Berkeley, in 1996. In 1993, he was a junior researcher at the Department of Electrical Systems and Automation of the
University of Pisa. In 1997, he joined the Parades g.e.i.e. research laboratory in Rome. His main research interests are in the field of hybrid systems, including reachability analysis, control synthesis and hybrid system discretization/relaxation; engine control in automotive applications; optimal control; man-machine interface for man-in-theloop systems; nonholonomic systems; variable structure and decentralized control.

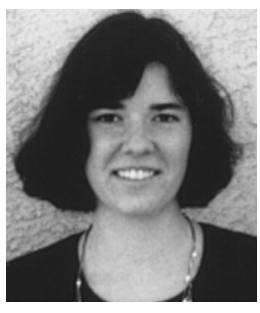

Lara S. Crawford received her A.B. in physics from Harvard University in 1991 She then attended the University of California at Berkeley, where she received her M.S. in electrical engineering sciences in 1993 and her Ph.D. in biophysics in 1998. She is currently employed as a research scientist at Optimal Synthesis Inc. Her research interests include learning control, nonlinear control, and biologically-inspired control.

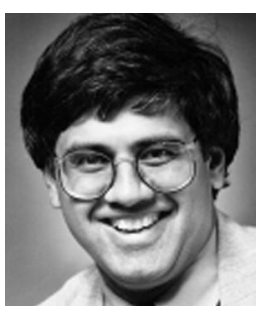

S. Shankar Sastry is a professor of Electical Engineering and Computer Sciences at the University of California, Berkeley. His research interests include Robotics, Nonlinear, Adaptive and Intelligent Control. In 1981 he received his Ph.D. from the EECS Department at University of California, Berkeley. He was the Gordon McKay Professor of Electrical Engineering and Computer Sciences, Harward University, 1994, Professore A Contratto, Unversita di Roma. Summer 1990, 1991, Scuola Normale di Pisa, 1992, Univ. di Pisa, 1994, Directeur Recherche, Center Nationale Recherche Scientifique (CNRS), Toulouse, France, Summer 1991, Visiting Fellow, Australian National University, Canberra, Summer 1985. He has been a Professor at the University of California, Berkeley, since 1988, and was an Assistant Professor, MIT, Cambridge, 1980-1982. He won the President of India Medal, 1977, IBM Faculty Development Grant, 1983, NSF Presidential Young Investigator Award, 1984, IEEE Student Best Paper Award, 1977, Eckmann Award of the American Control Council, 1990, M. A. (honoris causa, Harvard University, Cambridge, 1994, Fellow, IEEE, 1995. 